\title{
AMPK activation by Tanshinone IIA protects neuronal cells from oxygen-glucose deprivation
}

\author{
Yingfeng Weng ${ }^{1, *}$, Jixian Linn ${ }^{1, *}$, Hui Liu ${ }^{1, *}$, Hui Wu ${ }^{1}$, Zhimin Yan ${ }^{1}$ and Jing Zhao ${ }^{1}$ \\ ${ }^{1}$ Department of Neurology, Minhang Branch, Zhongshan Hospital, Fudan University, Shanghai, China \\ * Co-first authors \\ Correspondence to: Jing Zhao, email: zhaojinmhyy7@163.com \\ Zhimin Yan, email: yanzhiminmh@163.com \\ Keywords: OGDR; neuroprotection; Tanshinone IIA; AMP-activated protein kinase (AMPK); Ppmle
}

Received: October 27, $2017 \quad$ Accepted: December 01,2017 Published: December 17, 2017

Copyright: Weng et al. This is an open-access article distributed under the terms of the Creative Commons Attribution License 3.0 (CC BY 3.0), which permits unrestricted use, distribution, and reproduction in any medium, provided the original author and source are credited.

\section{ABSTRACT}

The current study tested the potential neuroprotective function of Tanshinone IIA (ThIIA) in neuronal cells with oxygen-glucose deprivation (ODG) and re-oxygenation (OGDR). In SH-SY5Y neuronal cells and primary murine cortical neurons, ThIIA pretreatment attenuated OGDR-induced viability reduction and apoptosis. Further, OGDR-induced mitochondrial depolarization, reactive oxygen species production, lipid peroxidation and DNA damages in neuronal cells were significantly attenuated by ThIIA. ThIIA activated AMP-activated protein kinase (AMPK) signaling, which was essential for neuroprotection against OGDR. AMPKa1 knockdown or complete knockout in SH-SY5Y cells abolished ThIIA-induced AMPK activation and neuroprotection against OGDR. Further studies found that ThIIA up-regulated microRNA-135b to downregulate the AMPK phosphatase Ppm1e. Notably, knockdown of Ppm1e by targeted shRNA or forced microRNA-135b expression also activated AMPK and protected SH-SY5Y cells from OGDR. Together, AMPK activation by ThIIA protects neuronal cells from OGDR. microRNA-135b-mediated silence of Ppm1e could be the key mechanism of AMPK activation by ThIIA.

\section{INTRODUCTION}

Ischemia-reperfusion shall cause damages to neurons $[1,2]$. In cultured neurons, oxygen-glucose deprivation (ODG) and re-oxygenation (OGDR) was applied to mimic the ischemia-reperfusion injuries [3-6]. Tanshinone IIA (ThIIA) is a phenanthrenequinone derivative from the Traditional Chinese Medicine Danshen [7, 8]. ThIIA has been utilized for the treatment of many diseases [7, 8]. The potential neuroprotective effect of ThIIA against OGDR is largely unknown.

Maintaining the physiological energy level in the neurons is vital for key cerebral behaviors. AMP-activated protein kinase (AMPK) is a key energy sensor [9-12]. Existing literatures have indicated that physiological AMPK also participates in brain development, neuronal polarization and other neuronal activities [9-12]. Additionally, deregulation of AMPK signaling could be involved in the development of neurodegenerative diseases [9-12].
Therefore, AMPK is emerging as a potential therapeutic target for neurodegenerative diseases [9-12]. Existing evidences have suggested that AMPK signaling is also important for cell survival, especially under certain stress conditions $[13,14]$. Here, we show that activation of AMPK is required ThIIA-mediated neuroprotection against OGDR.

\section{RESULTS}

Tanshinone IIA protects neuronal cells from oxygen glucose deprivation and re-oxygenation

The current study aims to understand the potential effect of Tanshinone IIA ("ThIIA") $[15,16]$ in neuron cells. SH-SY5Y is well-established human neuronal cell line [17-19]. The cell survival MTT assay results in Figure 1A showed that treatment with ThIIA alone at the tested concentrations $(0.1-10 \mu \mathrm{M})$ failed to change SH-SY5Y cell survival. In line with previous findings [3], SH-SY5Y 
cells exposure to oxygen glucose deprivation (OGD) and re-oxygenation (ODGR) resulted in significant cell viability ("MTT optic density, OD) reduction [3], which was largely inhibited by pre-treatment of ThIIA (Figure 1A). ThIIA displayed a dose-dependent manner in protecting SH-SY5Y cells from OGDR (Figure 1A). OGDR-induced SH-SY5Y cell death was reflected by the increase of lactate dehydrogenase (LDH) release to the medium (Figure 1B). Pre-treatment with $1-10 \mu \mathrm{M}$ of ThIIA significantly attenuated LDH release in OGDR-exposed SH-SY5Y cells (Figure 1B), again indicating its neuroprotective function. In the primary murine cortical neurons, OGDR exposure also resulted in dramatic cell death, causing LDH medium release (Figure 1C). Such effect was similarly inhibited by pre-treatment of ThIIA $(10 \mu \mathrm{M})$ (Figure 1C). Treatment with the ThIIA alone failed to change LDH content in the neuronal cells (Figure 1B and 1C). Thus, ThIIA protects neuronal cells from OGDR.

\section{Tanshinone IIA inhibits OGDR-induced neuronal cell apoptosis}

The potential effect of ThIIA on neuronal cell apoptosis was studied. As demonstrated, SH-SY5Y cells with OGDR exposure presented with significant increased activity of both Caspase-3 (Figure 2A) and Caspase-9 (Figure 2B). Furthermore, the content of histone-bound DNA (an apoptosis marker) was also elevated in OGDR-treated SH-SY5Y cells (Figure 2C). These results suggested apoptosis activation after OGDR exposure (Figure 2A-2C). Significantly, pre-treatment with ThIIA $(10 \mu \mathrm{M})$ largely attenuated OGDR-stimulated Caspase-3/-9 activation (Figure 2A and 2B) and histonebound DNA increase (Figure 2C) in SH-SY5Y cells. To further study cell apoptosis, Hoechst 33342 staining assay was performed. The nuclei with condensed or fragmented
Hoechst 33342 staining were labeled as the apoptotic nuclei $[20,21]$. Its ratio was quantified. As shown in Figure 2D, OGDR dramatically increased the apoptosis ratio in SH-SY5Y cells, which was largely inhibited by ThIIA $(10 \mu \mathrm{M})$ pretreatment (Figure $2 \mathrm{D})$. The very similar results were also observed in the primary murine cortical neurons, where ThIIA $(10 \mu \mathrm{M})$ pre-treatment efficiently suppressed OGDR-induced cell apoptosis (Hoechst assay, Figure 2E). It should be noted that treatment with ThIIA $(10 \mu \mathrm{M})$ alone failed to induce apoptosis in the neuronal cells (Figure 2A-2E). These results demonstrate that ThIIA inhibits OGDR-induced neuronal cell apoptosis.

\section{ThIIA attenuates OGDR-induced mitochondrial depolarization, ROS production, lipid peroxidation and DNA damages}

Mechanism insight studies have revealed that OGDR to neuronal cells will be followed by mitochondrial dysfunction, swelling and depolarization, ROS production, which will lead to lipid peroxidation, DNA damages and eventually cell apoptosis [3, 22-24]. In line with these findings, we found that OGDR exposure in SH-SY5Y neuronal cells also induced mitochondrial depolarization and ROS production, which were tested by increase of JC-1 green fluorescence intensity (Figure 3A) and 2',7'-dichlorofluorescein diacetate (DCFH-DA) fluorescence intensity (Figure 3B). Meanwhile, OGDR also caused lipid peroxidation (TBAR activity increase) (Figure 3C) and DNA damages ( $\mathrm{p}-\mathrm{H} 2 \mathrm{AX}$ increase, Figure 3D). Remarkably, such effects by OGDR were dramatically attenuated with ThIIA $(10 \mu \mathrm{M})$ pre-treatment (Figure $3 \mathrm{~A}-3 \mathrm{D})$. In the primary murine cortical neurons, ThIIA $(10 \mu \mathrm{M})$ similarly inhibited OGDR-induced ROS production (Figure 3E). Treatment with ThIIA $(10 \mu \mathrm{M})$ alone was ineffective (Figure $3 \mathrm{~A}-3 \mathrm{E})$.
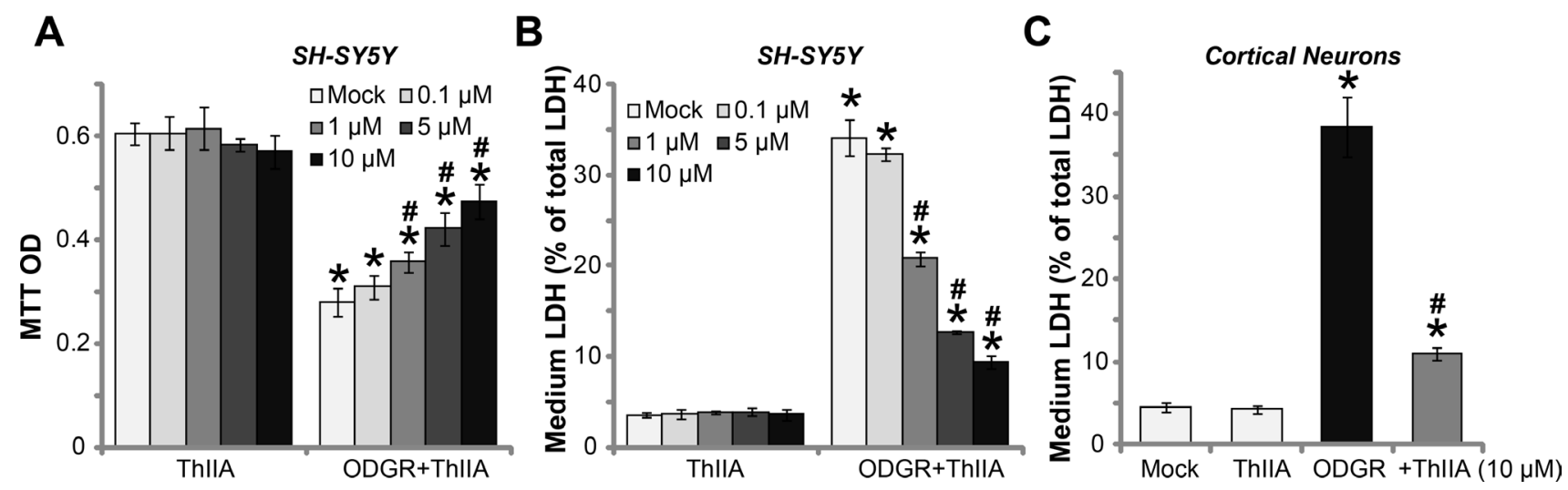

Figure 1: Tanshinone IIA protects neuronal cells from oxygen glucose deprivation and re-oxygenation. SH-SY5Y neuronal cells $(\mathbf{A}-\mathbf{B})$ or the primary murine cortical neurons $(\mathbf{C})$, pre-treated (for $30 \mathrm{~min}$ ) with indicated concentration of Tanshinone IIA ("ThIIA"), were exposed to oxygen glucose deprivation (OGD, for 4 hours) and re-oxygenation (for 24 hours, ODGR), cell survival was tested by MTT assay (optic density, or OD at $550 \mathrm{~nm}$ was recorded, (A); Cell death was tested by LDH release (B and C). "Mock" stands for normoxia treatment (Same for all Figures). Data were presented as mean $\pm \operatorname{SD}(n=5) .{ }^{*} P<0.05 v s$. "Mock" group. $P<0.05 v s$. ODGR only treatment (no ThIIA pre-treatment). Experiments in this figure were repeated four times, and similar results were obtained. 


\section{ThIIA activates AMPK signaling in neuronal cells}

As discussed, recent studies have suggested a prosurvival function of AMPK [25-27]. A number of AMPK activators were shown to protect cells from different stresses [28-31]. Previous studies have indicated that ThIIA could also activate AMPK signaling [32, 33]. We therefore examined AMPK signaling in ThIIA-treated neuronal cells. As shown in Figure 4A, in SH-SY5Y cells, ThIIA dose-dependently induced AMPK activation, which was reflected by increase of phosphorylations of AMPK $\alpha 1$ (at Thr-172) and its major downstream target acetyl-CoA carboxylase (ACC, at Ser-79). Meanwhile, AMPK activation was also evidenced by increase of AMPK $\alpha$ activity in ThIIA-treated SH-SY5Y cells (Figure 4B). The similar results were also obtained in ThIIAtreated primary murine cortical neurons, where AMPK/ ACC phosphorylations (Figure 4C) and AMPK $\alpha$ activity (Figure 4D) were significantly boosted. These results imply that ThIIA activates AMPK signaling in neuronal cells.

\section{Activation of AMPK is required for ThIIA-induced neuroprotection}

In order to study the link between ThIIA-induced neuroprotection and AMPK activation, shRNA method was applied to knockdown AMPKal. Two lentiviral AMPK $\alpha 1$ shRNAs, with non-overlapping sequences (S1 and S2, both from Dr. Lu's group [34, 35]), were added directly to cultured SH-SY5Y cells. Puromycin was then added to select stable cells. The quantitative real-time PCR (qRT-PCR) assay results confirmed that $A M P K \alpha 1 \mathrm{mRNA}$ level was dramatically downregulated in the stable SHSY5Y cells with AMPK $\alpha 1$ shRNA ("S1/2") (Figure 5A). Meanwhile, AMPK $\alpha 1$ protein expression and ThIIAinduced AMPK activation (reflected by p-ACC) were also largely inhibited (Figure 5B). The AMPK $\alpha 1$-silenced SH-SY5Y cells were more vulnerable to ODGR, showing increased viability reduction (Figure 5C) and LDH release (Figure 5D). Remarkably, ThIIA was almost ineffective in AMPKa1-silenced SH-SY5Y cells (Figure 5C and 5D). In the AMPK $\alpha 1$ shRNA SH-SY5Y cells, ThIIA treatment failed to protect against OGDR (Figure 5C and 5D).

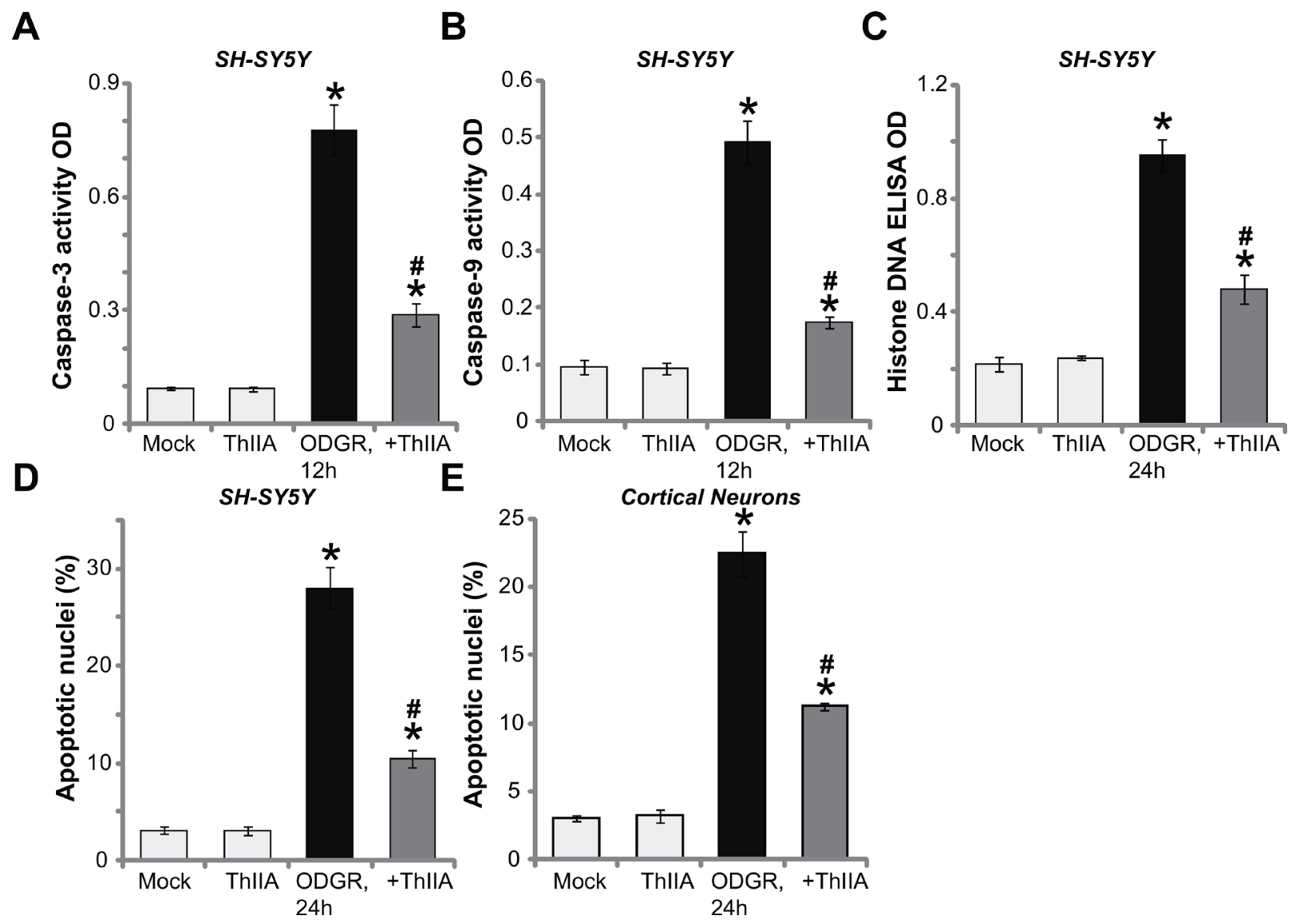

Figure 2: Tanshinone IIA inhibits OGDR-induced neuronal cell apoptosis. SH-SY5Y neuronal cells (A-D) or the primary murine cortical neurons (E), pre-treated (for $30 \mathrm{~min}$ ) with $10 \mu \mathrm{M}$ of Tanshinone IIA ("ThIIA"), were exposed to oxygen glucose deprivation (OGD, for 4 hours) and re-oxygenation (for applied hours, ODGR), the cell apoptosis assays mentioned in the text were performed. Data were presented as mean $\pm \mathrm{SD}(n=5) .{ }^{*} P<0.05$ vs. "Mock" group. ${ }^{\sharp} P<0.05 v s$. ODGR only treatment (no ThIIA pre-treatment). Experiments in this figure were repeated three times, and similar results were obtained. 
The results above implied that activation of AMPK might be required for ThIIA-induced neuroprotection against OGDR. To further support our hypothesis, CRISPR/Cas-9 method was applied to completely knockout AMPK $\alpha 1$. The CRISPR/Cas-9-AMPK $\alpha 1$ vector was introduced to SH-SY5Y cells, and stable cells were again established by puromycin selection. qRTPCR (Figure 5E) and Western blotting (Figure 5F) assay results confirmed complete depletion of AMPK $\alpha 1$ in the CRISPR/Cas-9-AMPK $\alpha 1$ ("AMPK $\alpha 1-K O ”)$ cells. ThIIAinduced ACC phosphorylation was completely blocked by CRISPR/Cas-9-AMPK $\alpha 1$ (Figure 5F). Similarly, OGDR exerted stronger cytotoxicity in AMPK $\alpha 1-\mathrm{KO}$ SH-SY $5 Y$ cells, as compared to cells with CRISPR/Cas-9 control ("CRISPR-C") (Figure 5G and 5H). More importantly, ThIIA-mediated anti-OGDR neuroprotection was almost completely nullified in the AMPK $\alpha 1-\mathrm{KO}$ SH-SY5Y cells (Figure $5 \mathrm{G}$ and $5 \mathrm{H}$ ). These results again confirm that activation of AMPK is required for ThIIA-induced neuroprotection.

\section{ThIIA increases miR-135b buts downregulates Ppm1e in neuronal cells}

Very recent research efforts have characterized $\mathrm{Ca}^{2+} /$ calmodulin-dependent protein kinase phosphatase (Ppm1e) as a key AMPK $\alpha 1$ phosphatase [31, 36, 37]. Ppm1e depletion or mutation could induce AMPK $\alpha 1$ phosphorylation and AMPK activation [31, 36, 37]. Here, the qRT-PCR assay results in Figure 6A demonstrated that ThIIA treatment caused a dramatic downregulation of Ppmle mRNA in SH-SY5Y cells. Meanwhile, Ppm1e protein expression was also reduced by ThIIA (Figure 6B). On the other hand, the Ppmle-targeting mRNA, miR$135 \mathrm{~b}$, was increased by ThIIA (Figure 6C). It should be noted that Ppmle $m R N A$ and protein expression as well as miR-135b expression were not changed by OGDR in SH-SY5Y cells (Figure 6A-6C).

The above results implied that ThIIA might possibly downregulate the AMPK $\alpha$ phosphatase Ppmle to activate AMPK. Next, a miR-135b expressing construct
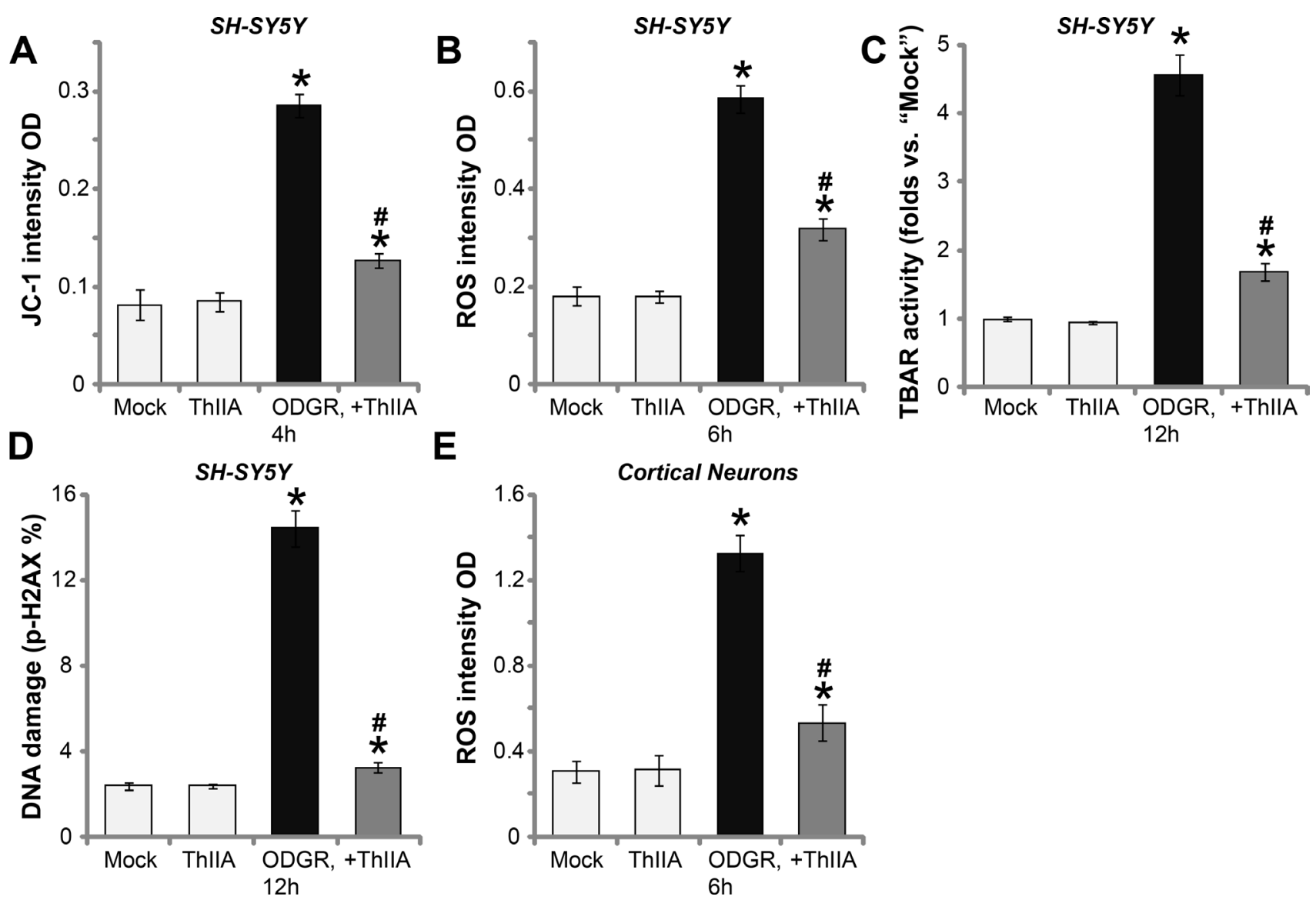

Figure 3: ThIIA attenuates OGDR-induced mitochondrial depolarization, ROS production, lipid peroxidation and DNA damages. SH-SY5Y cells (A-D) or the primary murine cortical neurons (E), pre-treated (for 30 min) with $10 \mu \mathrm{M}$ of Tanshinone IIA ("ThIIA"), were exposed to oxygen glucose deprivation (OGD, for 4 hours) and re-oxygenation (ODGR) for applied time, mitochondrial depolarization (A), ROS production (B and E), lipid peroxidation (C) and DNA damages (D) were tested by the assays mentioned in the text. Data were presented as mean $\pm \mathrm{SD}(n=5)$. ${ }^{*} P<0.05$ vs. "Mock" group. ${ }^{\sharp} P<0.05$ vs. ODGR only treatment (no ThIIA pre-treatment). Experiments in this figure were repeated three times, and similar results were obtained. 
("miR135b-Vec", a gift from Dr. Cui [31]) or the Ppm1e shRNA lentivirus ("sh-Ppm1e", also from Dr. Cui [31]) was introduced to SH-SY5Y cells. As shown in Figure 6D, forced expression of miR-135b-Vec or the Ppmle shRNA caused dramatic downregulation of Ppmle mRNA in SH-SY5Y cells.
Ppmle protein expression was almost completed depleted in stable cells with miR135b-Vec or the shRNA (Figure 6E). Notably, miR-135b and Ppmle shRNA both caused profound AMPK activation, which was evidenced by increased phosphorylations of AMPK $\alpha 1$ (Thr-172) and ACC (Ser-79)
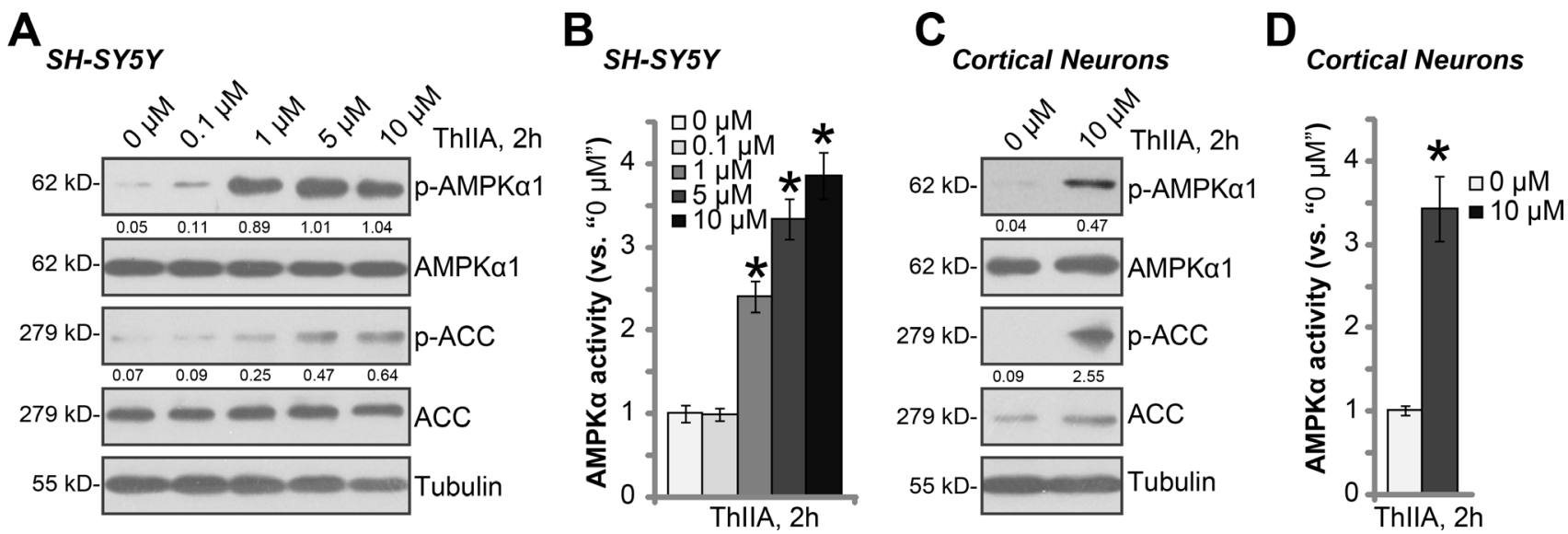

Figure 4: ThIIA activates AMPK signaling in neuronal cells. SH-SY5Y neuronal cells (A-B) or the primary murine cortical neurons (C-D) were treated with applied concentration of Tanshinone IIA ("ThIIA") for 2 hours, expressions of listed proteins in total cell lysates were shown (A and C); Relative AMPK $\alpha$ activity was also tested (B and D). AMPK $\alpha 1 / A C C$ phosphorylation was quantified (vs. total AMPKa1/ACC) (A and C). Data were presented as mean $\pm \mathrm{SD}(n=5)$. " $P<0.05$ vs. "0 $\mu \mathrm{M}$ " group (B and $\mathrm{D})$. Experiments in this figure were repeated three times, and similar results were obtained.
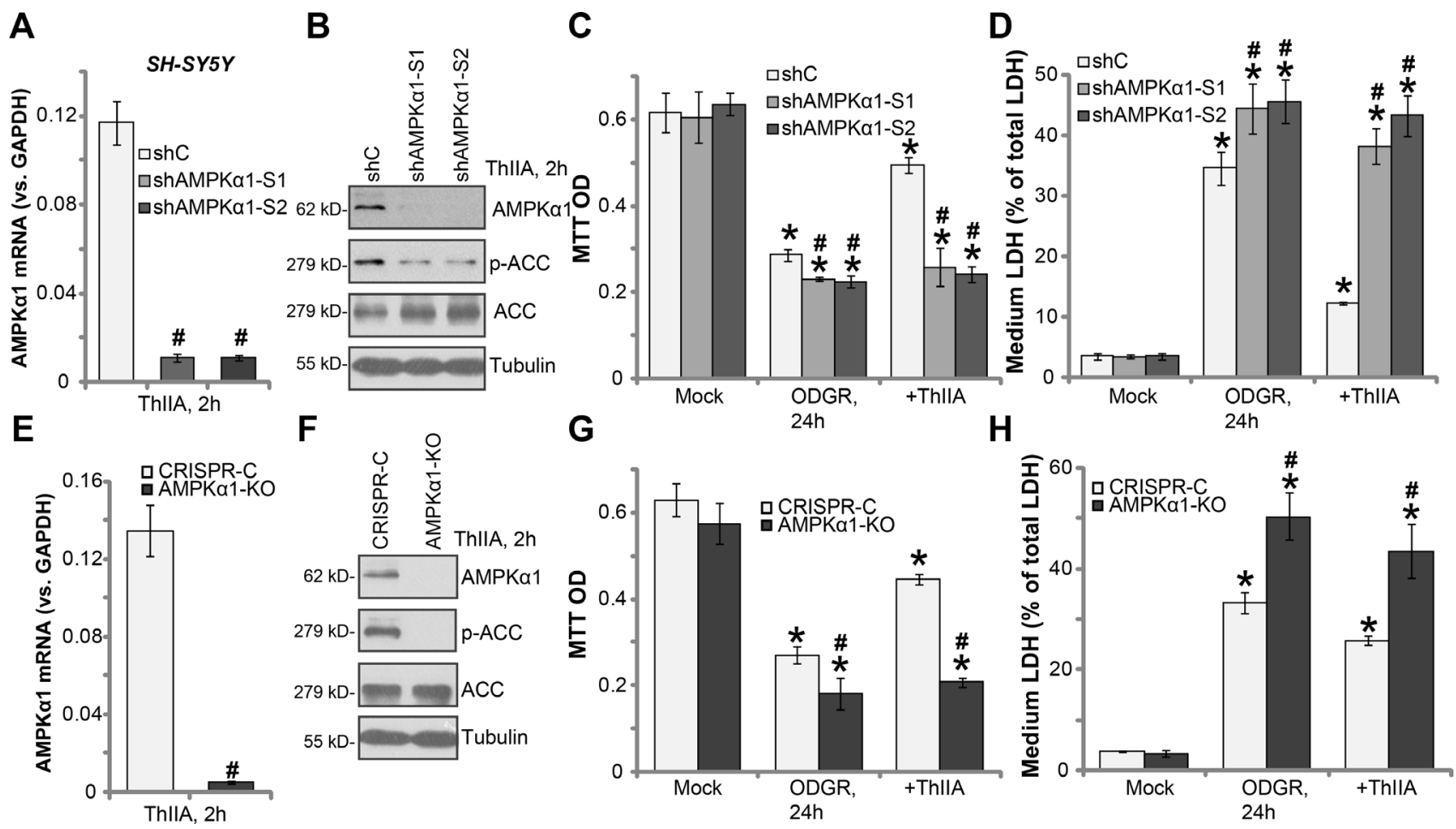

Figure 5: Activation of AMPK is required for ThIIA-induced neuroprotection. The stable SH-SY5Y cells, expressing AMPK $\alpha 1$ shRNA ("S1/2"), or scramble control shRNA ("shC") (A-D) as well as expressing CRISPR/Cas-9-AMPK $\alpha 1$ ("AMPK $\alpha 1-K O ")$ or CRISPR/Cas-9 control ("CRISPR-C"), were pre-treated with $10 \mu \mathrm{M}$ of Tanshinone IIA ("ThIIA"), with or without oxygen glucose deprivation (OGD, for 4 hours) and re-oxygenation (ODGR); AMPKal mRNA level was tested by qRT-PCR assay (A and E); Listed proteins in total cell lysates were shown (B and F); Cell survival and cell death were tested by MTT assay (C and $\mathbf{G})$ and LDH release assay (D and $\mathbf{H})$, respectively. Data were presented as mean $\pm \mathrm{SD}(n=5) .{ }^{*} P<0.05 v s$. "Mock" group. " $P<0.05 v s$. "shC" cells $(\mathrm{C}-\mathrm{D})$ or "CRISPR-C" cells $(\mathrm{G}$ and $\mathrm{H})$. Experiments in this figure were repeated three times, and similar results were obtained. 
(Figure 6E). qRT-PCR assay results in Figure 6F confirmed increased miR-135b expression in cells with the miR135bVec, which was not changed by Ppmle shRNA (Figure 6F).

Significantly, SH-SY5Y cells with miR-135b-Vec or the Ppmle shRNA were largely protected from OGDR, presenting with decreased viability reduction (Figure 6G) and reduced LDH release following OGDR (Figure 6H). Therefore, activation of AMPK by miR-135b or Ppm1e shRNA also protected SH-SY5Y cells from OGDR (Figure $6 \mathrm{G}$ and $6 \mathrm{H}$ ). Based on these results, we propose that ThIIA up-regulates miR-135b to downregulate Ppm1e, which possibly leads to AMPK $\alpha 1$ phosphorylation and AMPK activation, thus protecting neuronal cells from OGDR. Notably, miR-control vector ("miR-C") plus the non-sense control shRNA lentivirus ("sh-C") ("miR-C+sh-C") didn't exert above activities in SH-SY5Y cells (Figure 6D-6H).

\section{DISCUSSION}

Recent studies have verified a pivotal anti-oxidant function by activated AMPK. AMPK is important in maintaining NADPH balance [14]. Activated AMPK is shown to phosphorylate and inhibit ACC, causing decreased NADPH consumption [14]. Additionally, AMPK could also promote NADPH synthesis by fatty-acid oxidation [14]. Further, activated AMPK was also shown to activate Nrf2 signaling, a key anti-oxidant transcript factor [26, 38]. A recent study by Guo's group found that compound 13 (C13), an a1-selective AMPK activator, activated NADPH signaling to inhibit Dex-induced ROS production, therefore protecting osteoblastic cells [30]. Other AMPK activators showed similar anti-oxidant activity. Here, we showed that, in both SH-SY5Y neuronal cells and primary murine cortical neurons, ThIIA pretreatment attenuated OGDR-induced viability reduction and apoptosis. Further, OGDR-induced mitochondrial depolarization, ROS production, lipid peroxidation and DNA damages were also attenuated by ThIIA in neuronal cells. ThIIA activated AMPK signaling, which was required for it-mediated neuroprotection against OGDR. AMPK $\alpha 1$ knockdown (by targeted shRNA) or complete knockout (by CRISPR-Cas-9 method) abolished ThIIAinduced AMPK activation and neuroprotection.

Phosphorylation on Thr172 of catalytic $\alpha 1$ subunit of AMPK is required for its activation [39, 40]. The kinase phosphorylation of Thr172 of AMPK $\alpha 1$ has been studied extensively [41]. Several AMPK $\alpha$ kinases have been indentified, including liver kinase B1 (LKB1) [42], CaMKK (calcium/calmodulin-dependent protein kinase kinase) [43] and TAK1 (Transforming growth factor-b-activated kinase 1) [44, 45]. Recent studies have focused on the phosphatases that are responsible for dephosphorylating AMPK $\alpha 1$ at Thr172 [36]. Studies have indentified Ppmle, a $\mathrm{Ca}^{2+} /$ calmodulin-dependent protein kinase phosphatase [36], as well as PP2A catalytic subunit $[29,46]$, as possible AMPK phosphatases.
Inhibition or silence of the AMPK phosphatase has proven to be a fine strategy to induce AMPK activation. For instance, Cui's group demonstrated that miR-135b expression downregulated Ppm1e to activate AMPK signaling and protected osteoblastic cells [47]. microRNA429-mediated silence of PP2A catalytic subunit, another AMPK phosphatase, also induced AMPK activation and protected cells from oxidative stress [29]. In the current study, we demonstrated that ThIIA upregulated miR-135b and downregulated the AMPK phosphatase Ppm1e, which possibly led to AMPKa1 phosphorylation and AMPK activation. This could also be the primary mechanism of ThIIA-mediated neuroprotection against OGDR. Indeed, knockdown of Ppmle by targeted shRNA or forced expression of miR-135b also activated AMPK signaling and protected SH-SY5Y cells from OGDR, mimicking ThIIA functions.

\section{CONCLUSIONS}

Together, we propose that AMPK activation by ThIIA protects neuronal cells from OGDR. miR-135bmediated silence of Ppm1e could be the key mechanism of AMPK activation by ThIIA.

\section{METHODS}

\section{Cell culture}

Human neuronal cell line, SH-SY5Y, was purchased from the Cell Bank of Chinese Academy of Science (Shanghai, China). Cells were maintained in DMEM medium with $10 \%$ fetal bovine serum (FBS). Primary murine neurons were prepared from CA1 hippocampus of E14-E16 embryos of C57/B6 mouse. CA1 neurons $\left(100,000 \mathrm{cells} / \mathrm{cm}^{2}\right)$ were plated in serum-free neuron basal medium with 2\% B27 supplement and $2 \mathrm{mM}$ glutamine. All experimental procedures were approved by the Ethics Review Board and IACUC of authors' institutions.

\section{Reagents and antibodies}

Puromycin was purchased from Sigma (Shanghai, China). The Ppmle antibody was from Dr. Cui's group [31]. All other antibodies utilized in this study were obtained from Cell Signaling Tech (Danvers, MA). The cell culture reagents were obtained from Gibco Life Technologies (Carlsbad, CA).

\section{OGD/re-oxygenation (OGDR)}

Briefly, neuronal cells were first placed into an airtight chamber and equilibrated with a continuous flux of gas $\left(95 \% \mathrm{~N}_{2} / 5 \% \mathrm{CO}_{2}\right)$. The chamber was sealed and placed in an incubator for 4 hours (mimic oxygen glucose deprivation). Afterwards, the neuronal cells returned back to 
the complete medium and re-oxygenated. "Mock" control cells were placed in norm-oxygenated complete medium.

\section{MTT assay}

The survival of neuronal cells was evaluated by the routine 3-(4,5-dimethylthiazol-2-yl)-2,5-diphenyltetrazolium bromide (MTT, Sigma, Shanghai, China) according to the attached protocol. MTT optic density (OD) at $550 \mathrm{~nm}$ was recorded.

\section{LDH assay}

LDH release to the conditional medium reflects cell death intensity, which was examined via a commercial available two-step LDH detection kit (Promega, Shanghai, China). LDH content in the conditional medium was normalized to the total LDH.

\section{Apoptosis assay by enzyme-linked immunosorbent assay (ELISA)}

The Histone-DNA ELISA Detection Kit (Roche, Palo Alto, CA) was utilized to quantify cell apoptosis. Histone-DNA ELISA OD at $405 \mathrm{~nm}$ was recorded.

\section{Hoechst-33342 nuclei staining of apoptosis}

Following the treatment, neuronal cells were stained with Hoechst-33342 (Sigma, Shanghai, China). Non-apoptotic nuclei were with faint delicate chromatin blue staining, and nuclei with intensified/fragmented Hoechst-33342 staining were labeled as apoptotic nuclei. Apoptotic nuclei ratio was calculated, from at least 200 cells of 6 random views (1: 100).

\section{Caspase activity assay}

Following treatment, Caspase-3/-9 activity was tested via the Caspase-Glo-3/-9 activity assay kit (Promega, Nanjing, China), based on the attached protocols. The Caspase-3/-9 OD was recorded at $405 \mathrm{~nm}$ [48].

\section{Reactive oxygen species (ROS) detection}

Neuronal cells were stained with $1 \mu \mathrm{M}$ of DCFHDA (Invitrogen, Shanghai, China) for $30 \mathrm{~min}$. The DCF fluorescence signal was detected by a fluorescence microplate reader (Titertek Fluoroscan, Germany) at $550 \mathrm{~nm}$.

\section{Mitochondrial depolarization assay}

Briefly, neuronal cells were stained with JC-1 $(10 \mu \mathrm{g} / \mathrm{mL}$, Invitrogen, Shanghai, China) for $10 \mathrm{~min}$ at room temperature. JC-1 fluorescence intensity was examined using the fluorescence spectrofluorometer at $550 \mathrm{~nm}$ (Titertek Fluoroscan, Germany), testing membrane depolarization.

\section{Lipid peroxidation assay}

As described [49], cellular lipid peroxidation level was evaluated using the routine thiobarbituric acid reactive substances (TBAR) assay [50]. $30 \mu \mathrm{g}$ total cell lysates (per treatment) were mixed with $20 \%$ of acetic acid and thiobarbituric acid solution. After heating, the mixture was centrifuged, and the red pigment dye in the supernatant was examined by the microplate reader [50]. The TBAR activity, reflecting cellular lipid peroxidation level, was expressed as $\mathrm{nM}$ of malondialdehyde per mg protein.

\section{$\gamma-\mathrm{H} 2 \mathrm{AX}$ assay}

SH-SY5Y cells with the applied treatment were fixed and incubated with a mouse monoclonal anti-p- $\gamma-\mathrm{H} 2 \mathrm{AX}$ antibody (Cell Signaling Tech, Shanghai, China), followed by adding a FITC-conjugated anti-mouse secondary antibody (Santa Cruz). SH-SY5Y cells were then subjected to FACS assay to determine $\mathrm{p}-\gamma-\mathrm{H} 2 \mathrm{AX}$ percentage, reflecting DNA damage intensity [51].

\section{Western blotting assay}

The cell lysis buffer was purchased from Biyuntian (Wuxi, China). Quantified $30 \mu \mathrm{g}$ of proteins from total cell lysates were separated by SDS-page gels (10-12\%), which were transferred onto polyvinylidene difluoride (PVDF) blots (Millipore, Shanghai, China). After blocking, specific primary and corresponding secondary antibodies were added. The detection of the interested band was through the Enhanced chemiluminescence (ECL) reagents (Amersham Bioscience, Freiburg, Germany) and X-Ray film development. The ImageJ software was applied to quantify the intensity of each band.

\section{Quantitative real-time PCR}

As previously reported [52-54], TRIzol reagents (Invitrogen, Shanghai, China) were utilized to extract total cellular RNA. Quantitative real time-PCR ("qRT-PCR") assay was performed using the SYBR Green Master Mix Kit (Applied Biosystem) plus the ABI Prism 7600 Fast quantitative Real-Time PCR system (Foster City, CA). The mRNA primers for Ppmle, miR-135b-5p and GAPDH were provided by Dr. Cui's group [31]. mRNA primers for $A M P K \alpha 1$ were provided by $\mathrm{Dr}$. Lu [34, 35]. Melt curve analysis was applied to calculate product melting temperature. GAPDH was tested as the internal reference gene. The $2^{-\Delta \Delta C t}$ method was applied for the quantification $[52,53]$. 


\section{AMPKa activity assay}

Total cell lysates were immunoprecipitated with anti-pan-AMPK $\alpha 1$ antibody (Cell Signaling Tech, Shanghai, China). The AMPK $\alpha$ activity was determined in kinase assay buffer [55] plus AMP-[ $\left.\gamma-{ }^{32} \mathrm{P}\right]$ ATP mixture, and SAMS peptide (HMRSAMSGLHLVKRR) [55]. The reaction was terminated by spotting the reaction mixture. The radioactivity was measured with scintillation counter.

\section{shRNA and selection of stable cells}

The Ppmle lentiviral shRNA was provided by Dr. Cui [31]. The shRNA lentivirus was added to SH-SY5Y cells for 24 hours. The stable SH-SY5Y cells were then selected by puromycin $(1.0 \mu \mathrm{g} / \mathrm{mL})$ for $8-10$ days. The two lentivirus AMPK $\alpha 1$ shRNAs, with non-overlapping sequences (namely "S1" and "S2"), were provided by Dr. Lu's group [34, 35]. AMPK $\alpha 1$ shRNA lentivirus was added to SH-SY5Y cells, and stable cells were selected by puromycin. Expression of targeted protein in the stable cells was tested by Western blotting and/or qRT-PCR assays.

\section{CRISPR/Cas9-mediated knockout of AMPKa1}

The small guide RNA (sgRNA) targeting human AMPK $\alpha 1$ was based on the Optimized CRISPR Design application (http://crispr.mit.edu/), which was provided by Genepharm (Shanghai, China). AMPK $\alpha 1$ sgRNA was then inserted into the lenti-CRISPR plasmid (Addgene, Shanghai, China), which was transfected to SH-SY5Y cells. Puromycin was added to select stable cells. Complete AMPK $\alpha 1$ knockout was verified by Western blotting assay.

\section{microRNA-135b expression}

The lentiviral microRNA-135b (miR-135b) expression vector $(0.15 \mu \mathrm{g}$ construct per transfection), provided by Dr. Cui [31], was transfected to SH-SY5Y cells by Lipofectamine 2000 (Invitrogen, Shanghai, China). The stable SH-SY5Y cells were then selected by puromycin $(1.0 \mu \mathrm{g} / \mathrm{mL})$ for 8-10 days. Expression of miRNA-135b-5p in above cells was verified using qRT-PCR assay.

\section{Statistical analysis}

Data were presented as mean \pm standard deviation (SD). Statistics were analyzed by one-way ANOVA followed by a Scheffe' and Tukey Test (SPSS 16.0, Chicago, CA). $P<0.05$ means significant difference.

\section{Author contributions}

All authors listed in the current study carried out the experiments, participated in the design of the study and performed the statistical analysis, conceived of the study, and helped to draft the manuscript.

\section{CONFLICTS OF INTEREST}

The authors declare no conflicts of interest.

\section{FUNDING}

This study was supported by the National Natural Science Foundation of China (81572232) and by the Central Hospital of Shanghai Minxing District young student research foundation (2016 MHLC04, 2017MHLC10).

\section{REFERENCES}

1. Verklan MT. The chilling details: hypoxic-ischemic encephalopathy. J Perinat Neonatal Nurs. 2009; 23:59-68. https://doi.org/10.1097/01.JPN.0000346221. 48202.7e.

2. Allen CL, Bayraktutan U. Oxidative stress and its role in the pathogenesis of ischaemic stroke. Int J Stroke. 2009; 4:46170. https://doi.org/10.1111/j.1747-4949.2009.00387.x.

3. Zhao LP, Ji C, Lu PH, Li C, Xu B, Gao H. Oxygen glucose deprivation (OGD)/re-oxygenation-induced in vitro neuronal cell death involves mitochondrial cyclophilin-D/ P53 signaling axis. Neurochem Res. 2013; 38:705-13. https://doi.org/10.1007/s11064-013-0968-5.

4. Gu DM, Lu PH, Zhang K, Wang X, Sun M, Chen GQ, Wang Q. EGFR mediates astragaloside IV-induced Nrf2 activation to protect cortical neurons against in vitro ischemia/reperfusion damages. Biochem Biophys Res Commun. 2015; 457:391-7. https://doi.org/10.1016/j. bbrc.2015.01.002.

5. Almeida A, Delgado-Esteban M, Bolanos JP, Medina JM. Oxygen and glucose deprivation induces mitochondrial dysfunction and oxidative stress in neurones but not in astrocytes in primary culture. J Neurochem. 2002; 81:207-17.

6. Zhao H, Mitchell S, Ciechanowicz S, Savage S, Wang T, Ji X, Ma D. Argon protects against hypoxic-ischemic brain injury in neonatal rats through activation of nuclear factor (erythroid-derived 2)-like 2. Oncotarget. 2016; 7:25640-51. https://doi.org/10.18632/oncotarget.8241.

7. Chen $\mathrm{Z}, \mathrm{Xu} \mathrm{H}$. Anti-Inflammatory and Immunomodulatory Mechanism of Tanshinone IIA for Atherosclerosis. Evid Based Complement Alternat Med. 2014; 2014:267976. https://doi.org/10.1155/2014/267976.

8. Tian $\mathrm{XH}, \mathrm{Wu} \mathrm{JH}$. Tanshinone derivatives: a patent review (January 2006-September 2012). Expert Opin Ther Pat. 2013; 23:19-29. https://doi.org/10.1517/13543776.2013.7 36494.

9. Pineda-Ramirez N, Gutierrez Aguilar GF, EspinozaRojo M, Aguilera P. Current evidence for AMPK activation involvement on resveratrol-induced neuroprotection in cerebral ischemia. Nutr Neurosci. 2017; 1-19. https://doi. org/10.1080/1028415X.2017.1284361. 
10. Domise M, Vingtdeux V. AMPK in Neurodegenerative Diseases. EXS. 2016; 107:153-77. https://doi. org/10.1007/978-3-319-43589-3_7.

11. Liu YJ, Chern Y. AMPK-mediated regulation of neuronal metabolism and function in brain diseases. J Neurogenet. 2015; 29:50-8. https://doi.org/10.3109/01677063.2015.10 67203.

12. Pimentel GD, Ropelle ER, Rocha GZ, Carvalheira JB. The role of neuronal AMPK as a mediator of nutritional regulation of food intake and energy homeostasis. Metabolism. 2013; 62:171-8. https://doi.org/10.1016/j. metabol.2012.07.001.

13. Wang S, Song P, Zou MH. AMP-activated protein kinase, stress responses and cardiovascular diseases. Clin Sci (Lond). 2012; 122:555-73. https://doi.org/10.1042/CS20110625.

14. Jeon SM, Chandel NS, Hay N. AMPK regulates NADPH homeostasis to promote tumour cell survival during energy stress. Nature. 2012; 485:661-5. https://doi.org/10.1038/ nature11066.

15. Lin CY, Chang TW, Hsieh WH, Hung MC, Lin IH, Lai SC, Tzeng YJ. Simultaneous induction of apoptosis and necroptosis by Tanshinone IIA in human hepatocellular carcinoma HepG2 cells. Cell Death Dis. 2016; 2:16065. https://doi.org/10.1038/cddiscovery.2016.65.

16. Xu YM, Ding GH, Huang J, Xiong Y. Tanshinone IIA pretreatment attenuates ischemia/reperfusion-induced renal injury. Exp Ther Med. 2016; 12:2741-6. https://doi. org/10.3892/etm.2016.3674.

17. Zhang D, Zhang JJ, Liu GT. The novel squamosamide derivative FLZ protects against 6-hydroxydopamineinduced apoptosis through inhibition of related signal transduction in SH-SY5Y cells. Eur J Pharmacol. 2007; 561:1-6. https://doi.org/S0014-2999(06)01282-9.

18. Zhang L, Yu H, Sun Y, Lin X, Chen B, Tan C, Cao G, Wang Z. Protective effects of salidroside on hydrogen peroxide-induced apoptosis in SH-SY5Y human neuroblastoma cells. Eur J Pharmacol. 2007; 564:18-25. https://doi.org/S0014-2999(07)00170-7.

19. Wang K, Zhou F, Zhu X, Zhang K, Huang B, Zhu L. Neuroprotective properties of ciliary neurotrophic factor on retinoic acid (RA)-predifferentiated SH-SY5Y neuroblastoma cells. Folia Neuropathol. 2014; 52:121-27. https://doi.org/10.5114/fn.2014.43783.

20. Yu T, Li J, Sun H. C6 ceramide potentiates curcumininduced cell death and apoptosis in melanoma cell lines in vitro. Cancer Chemother Pharmacol. 2010; 66:999-1003. https://doi.org/10.1007/s00280-010-1374-1.

21. Li J, Hou N, Faried A, Tsutsumi S, Kuwano H. Inhibition of autophagy augments 5-fluorouracil chemotherapy in human colon cancer in vitro and in vivo model. Eur J Cancer. 2010; 46:1900-9. https://doi.org/10.1016/j.ejca.2010.02.021.

22. Zheng K, Sheng Z, Li Y, Lu H. Salidroside inhibits oxygen glucose deprivation (OGD)/re-oxygenation-induced H9c2 cell necrosis through activating of Akt-Nrf2 signaling. Biochem Biophys Res Commun. 2014; 451:79-85. https:// doi.org/10.1016/j.bbrc.2014.07.072.

23. Zheng K, Lu H, Sheng Z, Li Y, Xu B. Low-concentration of perifosine surprisingly protects cardiomyocytes from oxygen glucose deprivation. Biochem Biophys Res Commun. 2016; 469:753-60. https://doi.org/10.1016/j. bbrc.2015.12.014.

24. Lin JJ, Chang T, Cai WK, Zhang Z, Yang YX, Sun C, Li ZY, Li WX. Post-injury administration of allicin attenuates ischemic brain injury through sphingosine kinase 2: In vivo and in vitro studies. Neurochem Int. 2015; 89:92100. https://doi.org/10.1016/j.neuint.2015.07.022.

25. Varshney R, Gupta S, Roy P. Cytoprotective effect of kaempferol against palmitic acid-induced pancreatic betacell death through modulation of autophagy via AMPK/ mTOR signaling pathway. Mol Cell Endocrinol. 2017; 448:1-20. https://doi.org/10.1016/j.mce.2017.02.033.

26. Joo MS, Kim WD, Lee KY, Kim JH, Koo JH, Kim SG. AMPK Facilitates Nuclear Accumulation of Nrf2 by Phosphorylating at Serine 550. Mol Cell Biol. 2016; 36:1931-42. https://doi.org/10.1128/MCB.00118-16.

27. Yang M, Huang Y, Chen J, Chen YL, Ma JJ, Shi PH. Activation of AMPK participates hydrogen sulfide-induced cyto-protective effect against dexamethasone in osteoblastic MC3T3-E1 cells. Biochem Biophys Res Commun. 2014; 454:42-7. https://doi.org/10.1016/j.bbrc.2014.10.033.

28. Liu W, Mao L, Ji F, Chen F, Hao Y, Liu G. Targeted activation of AMPK by GSK621 ameliorates $\mathrm{H} 2 \mathrm{O} 2$-induced damages in osteoblasts. Oncotarget. 2017; 8:10543-52. https://doi.org/10.18632/oncotarget.14454.

29. Guo S, Chen C, Ji F, Mao L, Xie Y. PP2A catalytic subunit silence by microRNA-429 activates AMPK and protects osteoblastic cells from dexamethasone. Biochem Biophys Res Commun. 2017; 487:660-5. https://doi.org/10.1016/j. bbrc.2017.04.111.

30. Guo S, Mao L, Ji F, Wang S, Xie Y, Fei H, Wang XD. Activating AMP-activated protein kinase by an alpha1 selective activator compound 13 attenuates dexamethasoneinduced osteoblast cell death. Biochem Biophys Res Commun. 2016; 471:545-52. https://doi.org/10.1016/j. bbrc.2016.02.036.

31. Fan JB, Ruan JW, Liu W, Zhu LQ, Zhu XH, Yi H, Cui SY, Zhao JN, Cui ZM. miR-135b expression downregulates Ppmle to activate AMPK signaling and protect osteoblastic cells from dexamethasone. Oncotarget. 2016; 7:70613-22. https://doi.org/10.18632/oncotarget.12138.

32. Wu WY, Yan H, Wang XB, Gui YZ, Gao F, Tang XL, Qin YL, Su M, Chen T, Wang YP. Sodium tanshinone IIA silate inhibits high glucose-induced vascular smooth muscle cell proliferation and migration through activation of AMPactivated protein kinase. PLoS One. 2014; 9:e94957. https:// doi.org/10.1371/journal.pone.0094957. 
33. Hwang SL, Yang JH, Jeong YT, Kim YD, Li X, Lu Y, Chang YC, Son KH, Chang HW. Tanshinone IIA improves endoplasmic reticulum stress-induced insulin resistance through AMP-activated protein kinase. Biochem Biophys Res Commun. 2013; 430:1246-52. https://doi. org/10.1016/j.bbrc.2012.12.066.

34. Lu PH, Chen MB, Ji C, Li WT, Wei MX, Wu MH. Aqueous Oldenlandia diffusa extracts inhibits colorectal cancer cells via activating AMP-activated protein kinase signalings. Oncotarget. 2016; 7:45889-900. https://doi.org/10.18632/ oncotarget.9969.

35. Chen MB, Jiang Q, Liu YY, Zhang Y, He BS, Wei MX, Lu JW, Ji Y, Lu PH. C6 ceramide dramatically increases vincristine sensitivity both in vivo and in vitro, involving AMP-activated protein kinase-p53 signaling. Carcinogenesis. 2015; 36:1061-70. https://doi.org/10.1093/ carcin/bgv094.

36. Voss M, Paterson J, Kelsall IR, Martin-Granados C, Hastie CJ, Peggie MW, Cohen PT. Ppm1E is an in cellulo AMP-activated protein kinase phosphatase. Cell Signal. 2011; 23:114-24. https://doi.org/10.1016/j. cellsig.2010.08.010.

37. Li P, Fan JB, Gao Y, Zhang M, Zhang L, Yang N, Zhao X. miR-135b-5p inhibits LPS-induced TNFalpha production via silencing AMPK phosphatase Ppm1e. Oncotarget. 2016; 7:77978-86. https://doi.org/10.18632/ oncotarget.12866.

38. Zimmermann K, Baldinger J, Mayerhofer B, Atanasov AG, Dirsch VM, Heiss EH. Activated AMPK boosts the Nrf2/ HO-1 signaling axis - A role for the unfolded protein response. Free Radic Biol Med. 2015; 88:417-26. https:// doi.org/10.1016/j.freeradbiomed.2015.03.030.

39. Hardie DG, Ross FA, Hawley SA. AMPK: a nutrient and energy sensor that maintains energy homeostasis. Nat Rev Mol Cell Biol. 2012; 13:251-62. https://doi.org/10.1038/ nrm3311.

40. Mihaylova MM, Shaw RJ. The AMPK signalling pathway coordinates cell growth, autophagy and metabolism. Nat Cell Biol. 2011; 13:1016-23. https://doi.org/10.1038/ ncb2329.

41. Hardie DG. AMP-activated/SNF1 protein kinases: conserved guardians of cellular energy. Nat Rev Mol Cell Biol. 2007; 8:774-85. https://doi.org/10.1038/nrm2249.

42. Shaw RJ, Kosmatka M, Bardeesy N, Hurley RL, Witters LA, DePinho RA, Cantley LC. The tumor suppressor LKB1 kinase directly activates AMP-activated kinase and regulates apoptosis in response to energy stress. Proc Natl Acad Sci U S A. 2004; 101:3329-35. https://doi. org/10.1073/pnas.0308061100.

43. Jensen TE, Rose AJ, Jorgensen SB, Brandt N, Schjerling P, Wojtaszewski JF, Richter EA. Possible CaMKK-dependent regulation of AMPK phosphorylation and glucose uptake at the onset of mild tetanic skeletal muscle contraction. Am J Physiol Endocrinol Metab. 2007; 292: E1308-17. https:// doi.org/10.1152/ajpendo.00456.2006.

44. Chen Z, Shen X, Shen F, Zhong W, Wu H, Liu S, Lai J. TAK1 activates AMPK-dependent cell death pathway in hydrogen peroxide-treated cardiomyocytes, inhibited by heat shock protein-70. Mol Cell Biochem. 2013; 377:35-44. https://doi.org/10.1007/ s11010-013-1568-z.

45. Herrero-Martin G, Hoyer-Hansen M, Garcia-Garcia C, Fumarola C, Farkas T, Lopez-Rivas A, Jaattela M. TAK1 activates AMPK-dependent cytoprotective autophagy in TRAIL-treated epithelial cells. EMBO J. 2009; 28:677-85. https://doi.org/10.1038/emboj.2009.8.

46. Wu Y, Song P, Xu J, Zhang M, Zou MH. Activation of protein phosphatase $2 \mathrm{~A}$ by palmitate inhibits AMP-activated protein kinase. J Biol Chem. 2007; 282:9777-88. https:// doi.org/10.1074/jbc.M608310200.

47. Li ZW, Zhu YR, Zhou XZ, Zhuo BB, Wang XD. microRNA-135b expression silences Ppm1e to provoke AMPK activation and inhibit osteoblastoma cell proliferation. Oncotarget. 2017; 8:26424-33. https://doi. org/10.18632/oncotarget.15477.

48. Zou Y, Ge M, Wang X. Targeting PI3K-AKT-mTOR by LY3023414 inhibits human skin squamous cell carcinoma cell growth in vitro and in vivo. Biochem Biophys Res Commun. 2017; 490:385-92. https://doi.org/10.1016/j. bbrc.2017.06.052.

49. Kwon MY, Park E, Lee SJ, Chung SW. Heme oxygenase-1 accelerates erastin-induced ferroptotic cell death. Oncotarget. 2015; 6:24393-403. https://doi.org/10.18632/ oncotarget.5162.

50. Cortizo AM, Bruzzone L, Molinuevo S, Etcheverry SB. A possible role of oxidative stress in the vanadium-induced cytotoxicity in the MC3T3E1 osteoblast and UMR106 osteosarcoma cell lines. Toxicology. 2000; 147:89-99. https://doi.org/S0300483X00001815.

51. Ewald B, Sampath D, Plunkett W. H2AX phosphorylation marks gemcitabine-induced stalled replication forks and their collapse upon S-phase checkpoint abrogation. Mol Cancer Ther. 2007; 6:1239-48. https://doi.org/10.1158/ 1535-7163.MCT-06-0633.

52. Chen MB, Yang L, Lu PH, Fu XL, Zhang Y, Zhu YQ, Tian Y. MicroRNA-101 down-regulates sphingosine kinase 1 in colorectal cancer cells. Biochem Biophys Res Commun. 2015; 463:954-60. https://doi.org/10.1016/j. bbrc.2015.06.041.

53. Chen MB, Wei MX, Han JY, Wu XY, Li C, Wang J, Shen W, $\mathrm{Lu}$ PH. MicroRNA-451 regulates AMPK/mTORC1 signaling and fascin 1 expression in HT-29 colorectal cancer. Cell Signal. 2014; 26:102-9. https://doi.org/10.1016/j. cellsig.2013.07.017. 
54. Chen MB, Zhou ZT, Yang L, Wei MX, Tang M, Ruan TY, Xu JY, Zhou XZ, Chen G, Lu PH. KU-0060648 inhibits hepatocellular carcinoma cells through DNA-PKcsdependent and DNA-PKcs-independent mechanisms. Oncotarget. 2016; 7:17047-59. https://doi.org/10.18632/ oncotarget. 7742 .
55. Lee M, Hwang JT, Lee HJ, Jung SN, Kang I, Chi SG, Kim SS, Ha J. AMP-activated protein kinase activity is critical for hypoxia-inducible factor-1 transcriptional activity and its target gene expression under hypoxic conditions in DU145 cells. J Biol Chem. 2003; 278:39653-61. https://doi.org/10.1074/jbc.M306104200. 